\title{
Iridocyclo-retraction in narrow-angle glaucoma
}

\author{
M. M. KRASNOV \\ Second Medical Institute, Moscow, USSR
}

No specific way has so far been found to treat the organic (synechial) closure of the anterior chamber angle. Iridectomy relieves the pupillary block but the iris root remains fixed to the filtering zone. Cyclodyalisis is as a rule useless, being followed by immediate re-adherence.

A new surgical procedure which has been developed is described in this paper as "iridocyclo-retraction". The iris root is forced by two (or more) interposing autoimplants of scleral tissue (Fig. I).

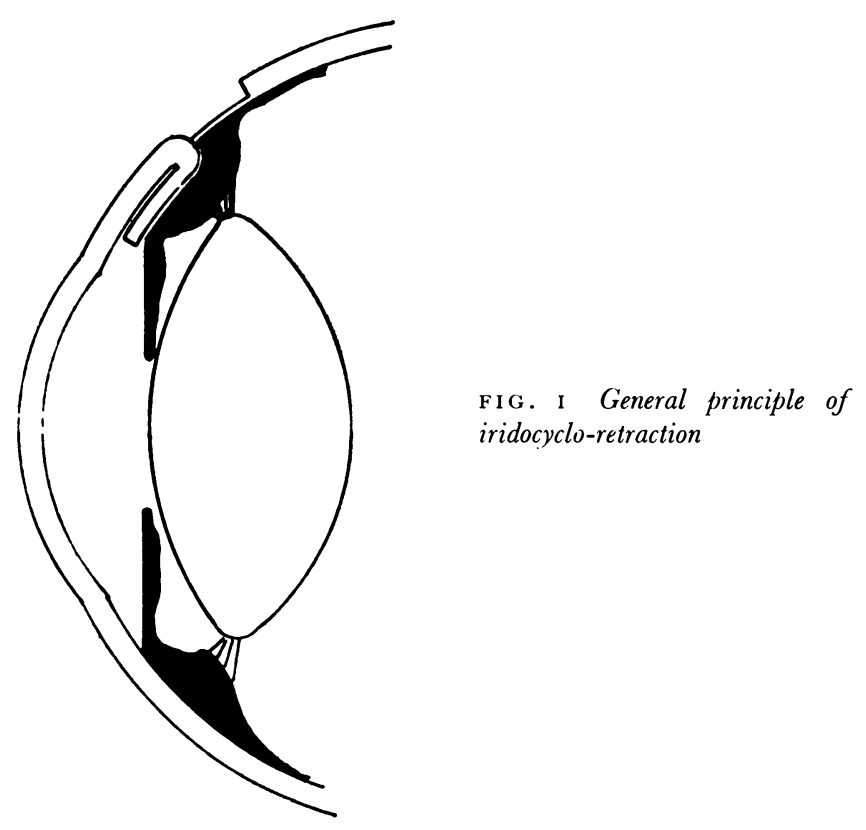

So-called "setons" of various substances (plastics, etc.) have already been tried to keep the cyclodialysis cleft open. The new procedure has two important features:

(i) It aims to restore the normal access of the aqueous to the outflow pathways (rather than to provide a new connection between the anterior chamber and the suprachoroidal space, as in cyclodialysis).

(ii) It is autoplastic. 


\section{Preliminary studies}

Experiments on rabbits (Lakomkin, 1970) have shown that the procedure is well tolerated by the eye, and that it achieves its object of restoring access to the normal outflow system.

Histological studies showed that additional outflow routes might be formed along the autoimplants, and endothelium-lined slits have, in fact, been found to form. Connection with the suprachoroidal space may occasionally result, but this is not the main purpose of the procedure.

An exceptionally interesting case was observed by Shmeleva (1970); a patient who had undergone iridocyclo-retraction died a month later from an unrelated cause. It was found at the post mortem examination that the angle was open, whereas before the operation it was nearly closed.

Surgical technique (Fig. 2, opposite).

Local anaesthesia is achieved by a retrobulbar injection of $2 \mathrm{ml}$. procaine 2 per cent. solution; another $2 \mathrm{ml}$. of the same solution is injected along the superior rectus muscle and (on withdrawal of the needle) under the conjunctiva.

An incision is made in the conjunctiva $8 \mathrm{~mm}$. from the limbus, usually between I I and I o'clock, and the sclera is exposed almost to the limbus. The rectus muscle is clamped by a small haemostat to diminish the blood supply to the operated region.

Two small $(2 \times 4 \mathrm{~mm}$.) pedicles are then outlined by nonperforating incisions of the sclera (Fig. 2$)$, 4-8 $\mathrm{mm}$. from the limbus. The two areas should be about $5 \mathrm{~mm}$. apart; the width of the muscle insertion is a convenient criterion.

Next the sclera is laminated inside the outlined zones, leaving approximately one-third of its thickness below. Anteriorly $(4 \mathrm{~mm}$. from the limbus) the two pedicles remain attached to the sclera.

Cuts are then made at the base of the flaps through the remaining layers into the suprachoroidal space, and bleeding points are cauterized.

A thin cyclodialysis spatula is introduced beneath the sclera into the anterior chamber angle. The roots of the iris and the anterior part of the ciliary body are gently separated from the sclera between the two flaps. A strong pressure on the eye is exerted for about I min. to prevent bleeding into the anterior chamber.

The most delicate (and very important) step is the introduction of the scleral pedicles into the anterior chamber angle; for this the following technique can be recommended:

A thin round needle (as used in vascular surgery) is grasped by the needleholder, and the anterior half of the needle $(5 \mathrm{~mm}$.) is straightened out. The free end of the pedicle is picked up by the needle point and then pulled forward under the sclera. The attachment of the pedicles to the sclera prevents them from being pushed too far in the anterior chamber (their ends should be at the limbus). Their position can easily be corrected by a thin spatula. When the needle is withdrawn it slips off leaving the flaps in place.

We have also tried "free" autoimplants (using scleral tissue or auricular cartilage), but their placing is more difficult.

Air is injected into the anterior chamber, care being taken not to cover the pupillary region by the bubble (which may cause an air block). The conjunctival incision is closed by an uninterrupted suture.

COMPLICATIONS

The procedure is essentially simple, but requires attention given to minute details. The same complications may arise as in cyclodialysis: iridodialysis, perforation of the ciliary body, detachment of Descemet's membrane, etc. They result from technical errors and need not be discussed here. 
(A)
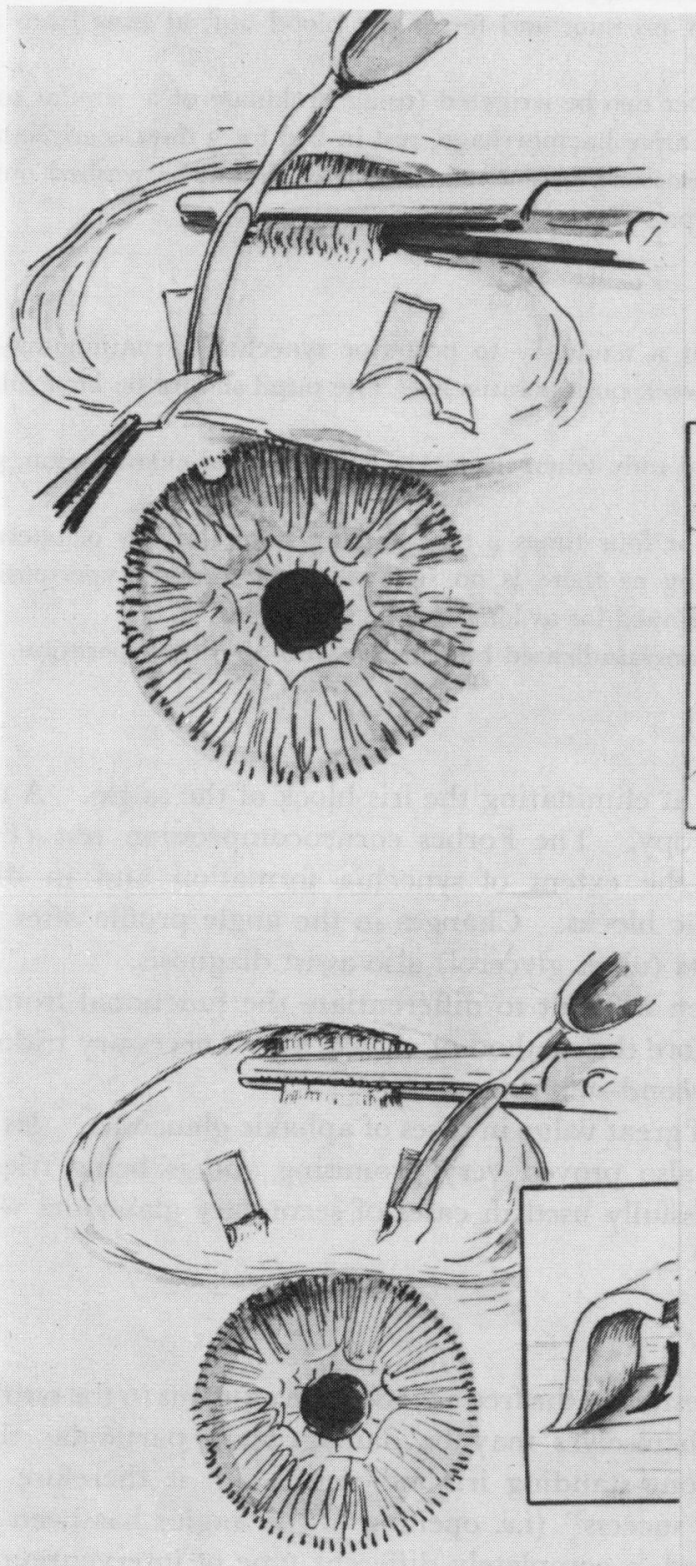

(c)
(B)
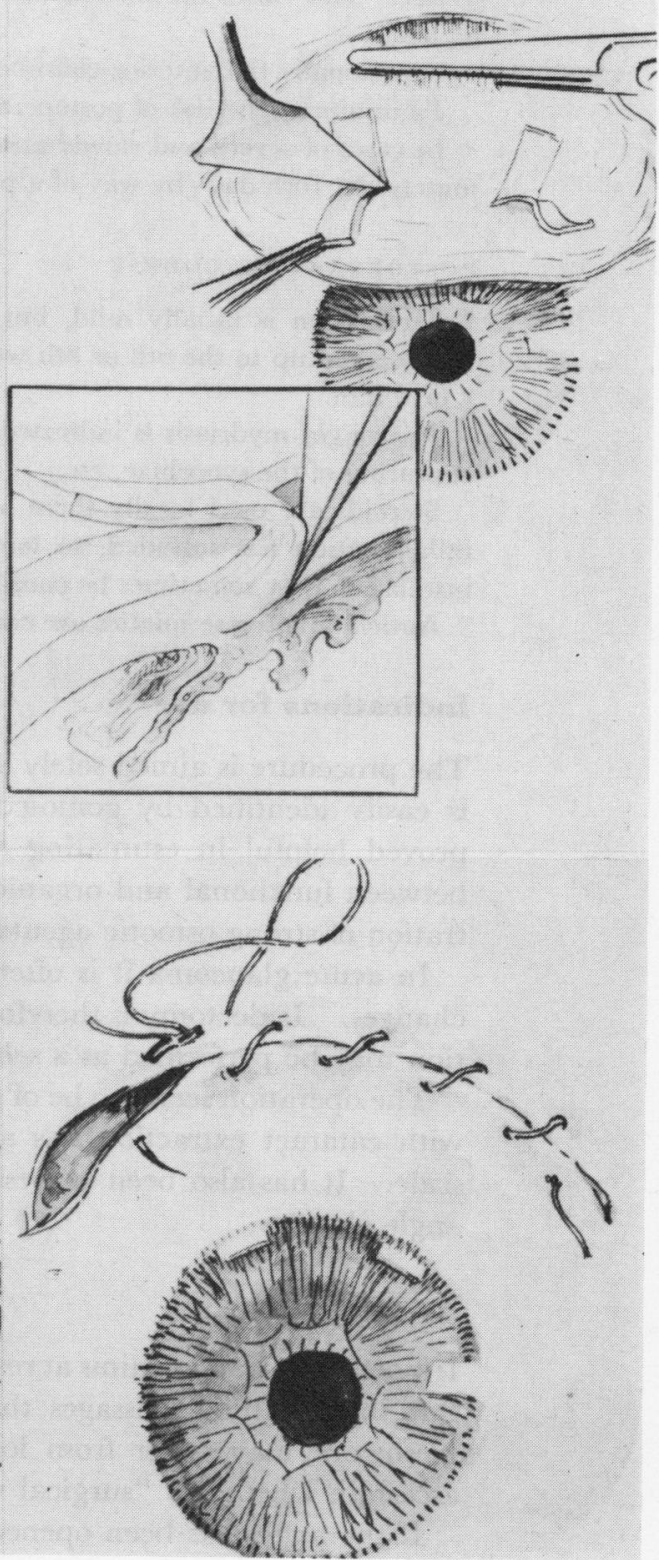

(D)

FIG. 2 Technique of iridocyclo-retraction $(a, b, c, d)$. For explanation see text

The greatest care should be taken to avoid bleeding into the anterior chamber, which predisposes to synechia formation and may nullify the effect of the operation.

The critical moment is that of separation of the iris root from the ciliary body. A sufficiently strong pressure (for instance, by a cotton swab) on the eye is a routine and effective preventive measure. In the process of separation the 3 and 9 o'clock meridians are to be avoided (because of the large vessels in the vicinity). 
If, in spite of all precautions, blood appears in the anterior chamber, air should be quickly injected. This raises the intracoular pressure and forces the blood out, at least from the pupillary region.

Occasionally the anterior chamber can be irrigated (using urokinase or a similar solution).

To minimize the risk of postoperative haemorrhage, rest in bed for 3 days is advisable.

In cases of severe and slowly-absorbing hyphaema, the latter should be washed out on approximately the roth day (by way of a paracentesis).

POSTOPERATIVE GOURSE

Inflammation is usually mild, but a tendency to posterior synechia formation may occasionally कै be observed up to the 6 th or 8 th week postoperatively. The pupil should be kept dilated to about $\vec{\circ}$ 5 or $6 \mathrm{~mm}$.

A stronger mydriasis is indicated only when necessary for periodical examination, prevention, or $\mathscr{O}^{\omega}$ rupturing of the synechiae, etc.

Steroids are used locally three or four times a day starting from the day of operation. When of inflamination has subsided, so long as there is no sign of corticosteroid hypertension, the daily or instillation may sometimes be continued for as long as 2 or 3 months.

Anticholinesterase miotics are contraindicated both before and after the operation.

\section{Indications for use}

The procedure is aimed solely at eliminating the iris-block of the angle. A narrow angle is easily identified by gonioscopy. The Forbes corneocompression test (Forbes, 1966) $\vec{\varphi}$ proved helpful in estimating the extent of synechia formation and in differentiating.between functional and organic blocks. Changes in the angle profile after the administration of strong osmotic agents (urea, glycerol) also assist diagnosis.

In acute glaucoma it is often difficult to differentiate the functional from the organic changes. Iridectomy is therefore the method of choice, and if necessary iridocyclo-retrac- $\stackrel{\infty}{\mathbb{D}}$ tion may be performed as a second-step procedure

The operation seems to be of great value in cases of aphakic glaucoma. Its combination with cataract extraction has also proved very promising and is being tried on a wide $\vec{F}$ scale. It has also been successfully used in cases of secondary glaucoma with synechial angle closure.

\section{Re-operation}

Iridocyclo-retraction aims at restoring the free access of the aqueous to the outflow channels. But these outflow passages themselves may be damaged; in particular the trabecularo meshwork may suffer from long-standing iris adherence. It is therefore important to ascertain whether a "surgical success" (i.e. opening of the angle) has been achieved.

If the angle has been opened, a completely different type of intervention is needed on N the intramural outflow passages. This may be trabeculectomy (after Cairns), trabecul- N otomy, or other methods. This second-stage procedure has to be carried out over the area N of the opened angle (i.e. between the implants). On the other hand, if the angle stillg remains closed, the object of the subsequent procedure is the same as that of the first.

Re-adherence of the iris root between the implants may sometimes follow if they have been placed too far apart; the space between them should not exceed $5 \mathrm{~mm}$., and if this? happens iridocyclo-retraction should be repeated.

Separation of the iris root is carried out in the same region and a third pedicle is introduced between the former two (Fig. 3, opposite). 


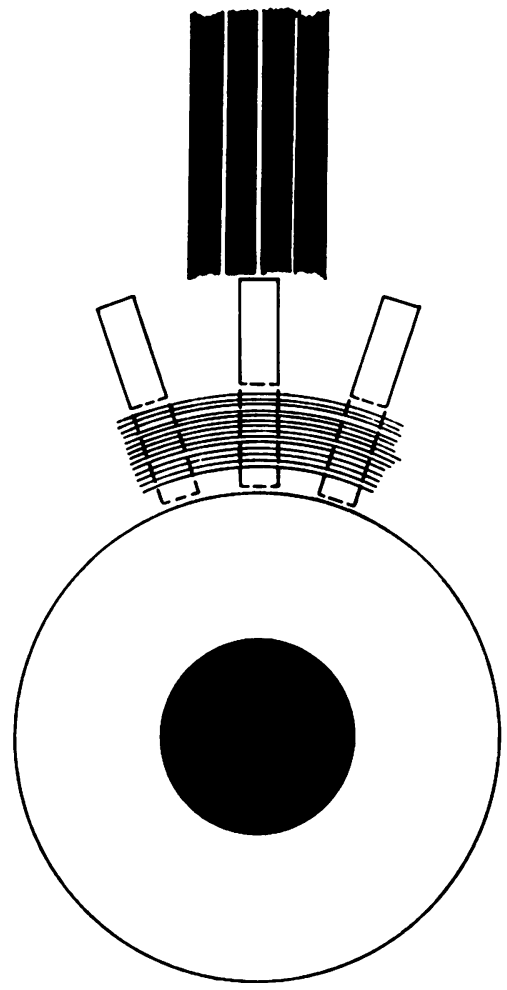

FIG. 3 "Three-point" version of iridocyclo-retraction. The black bars indicate the position of a rectus muscle (usually the superior). The area of opening of the anterior chamber angle is plotted by the concentric lines

FIG. 4 Diagram illustrating gonioscopic view after iridocycloretraction. The angle is opened between the autoimplants

Sometimes, when it appears that the thrust behind the iris is too strong, this "threepoint" iridocyclo-retraction is done as a primary procedure.

\section{Results}

I 4 I iridocyclo-retraction operations have so far been performed (including 23 in cases of aphakia). It is now commonly used in everyday practice and the maximum follow-up is 3 years. The anterior chamber angle was successfully opened after the first procedure in 128 of 141 eyes, and a second "three-point" iridocyclo-retraction resulted in opening the angle in seven of the remaining thirteen cases.

Ocular hypertension recurred in 22 cases after the angle had been opened, and a second operation (trabeculectomy, sinusotrabeculotomy) was followed by reduction of the ocular tension to normal in fifteen of these cases.

The tension was controlled after the first procedure in about 80 per cent. of cases, and the second operation brought the success rate to about 90 per cent. A longer followup is needed to confirm these data.

In one case a choroidal detachment developed after the operation, but this disappeared spontaneously in a few days.

A long-standing hypotony observed in four cases seemed to hasten pre-existing cataractous changes.

The gonioscopic picture after an iridocyclo-retraction operation is very characteristic (Fig. 4). The autoimplants are clearly seen to protrude into the anterior chamber angle from behind. When the procedure has been successful, the angle is opened between the pedicles. 


\section{Discussion}

It will be remembered that this iridocyclo-retraction operation forms part of a system of nonfistulizing glaucoma surgery (Krasnov, I968, 1969). This has been described elsewhere and is summarized in the Table.

Table Nonfistulizing glaucoma surgery

\begin{tabular}{|c|c|}
\hline Causes of intraocular hypertension & Surgery \\
\hline $\begin{array}{ll}\text { Iris-block (angular) retention } & \text { (a) Functional } \\
& \text { (b) Organic (synechial) }\end{array}$ & $\begin{array}{l}\text { Iridectomy } \\
\text { Iridocyclo-retraction }\end{array}$ \\
\hline Pretrabecular obstruction by foreign tissue & Goniotomy \\
\hline "Trabecular" retention & $\begin{array}{l}\text { Trabeculotomy or } \\
\text { trabeculectomy }\end{array}$ \\
\hline "Intrascleral" retention & Sinusotomy \\
\hline "Hypersecretion" glaucoma & Cycloanaemization \\
\hline Combination of causes & Combined procedures \\
\hline
\end{tabular}

If used alone, iridocyclo-retraction is indicated for iris-block (narrow-angle) glaucoma only. If iris-block is combined with intramural (usually, trabecular) damage, $\vec{\varphi}$ iridocyclo-retraction can be combined with the appropriate procedure in the above system. -

The operation is not recommended for wide-angle glaucoma, because a hypertensive effect may ensue owing to drainage into the suprachoroidal space (as in cyclodialysis).

Although iridocyclo-retraction is primarily aimed at synechial angle closure, it is also quite efficient in cases of functional iris-block. The choice between iridectomy and irido- $\frac{\circ}{\infty}$ cyclo-retraction is therefore of clinical importance. Iridectomy has traditionally been the method of choice. It is simple and safe, but it is usually insufficient in the presence $\frac{9}{3}$ of the extensive goniosynechiae which commonly occur in narrow-angle glaucoma. In short, iridectomy is promising only in certain cases of glaucoma due to anomalies of the angle, whereas iridocyclo-retraction is nearly always so.

The mechanics of the hypertensive action of iridocyclo-retraction deserve some consideration. Several factors may be involved: opening of the angle, drainage into the suprachoroidal space, new-formed outflow channels along the autoimplants, and the inhibition of aqueous production by the ciliary body.

The paramount question is whether the effect is due to an increase in outflow or to a

decrease in aqueous formation.
Repeated tonographic investigations (Lakomkin, 1970) over a 12-month period after sixteen uneventful iridocyclo-retractions showed the following data:

Before surgery:

$$
\text { Average } \mathrm{G}=0 \cdot \mathrm{Io} \pm 0 \cdot 0 \mathrm{I} ; \mathrm{F}=\mathrm{I} \cdot 2 \pm 0 \cdot \mathrm{I} 8 ; \mathrm{P}_{0} / \mathrm{C} 34^{2} \pm 76
$$

After surgery:

$$
\text { Average } \mathrm{C}=0.24 \pm 0.01 ; \mathrm{F}=0.9 \pm 0.12 ; \mathrm{P}_{0} / \mathrm{C} 55 \pm 4
$$

This suggests than an improvement in the outflow facility is the principle factor, though some reduction in aqueous formation may also result. 
The conventional procedures are not always satisfactory in cases of synechial angle closure. Even the traditional fistulizing surgery is here less effective. Iridencleisis though widely used, is actually one of the most injurious procedures in glaucoma surgery, and $I$ have practically abandoned its use.

\section{Summary}

A new surgical prodecure (iridocyclo-retraction) has been developed for narrow-angle glaucoma, especially in cases with synechial iris closure. The iris root is forced back from the filtering zone and kept back by two (sometimes three) interposing autoimplants of scleral tissue. The operation has been successfully tried in I4I cases during a 3-year period. Experiments on rabbits have shown the method to be based on sound principles. Iridocyclo-retraction has substantial advantages over iridectomy and iridencleisis and deserves a wider clinical use.

\section{References}

FORBES, M. (1966) Arch. Ophthal. (Chicago), 76, 488

LAKomkin, v. I. (1970) Vestn. Oftal., No. 2, p. 48

KRASNOV, м. м. (1968) Ibid., No. 6, p. 58

(1969) Amer. F. Ophthal., 67, 857

shmeleva, v. v. (1970) Personal communication 